\title{
MODELING SUNSPOT AND STARSPOT DECAY BY TURBULENT EROSION
}

\author{
YURi E. LiTVINENKo ${ }^{1}$ aNd M. S. WheATLAND ${ }^{2}$ \\ ${ }^{1}$ Department of Mathematics, University of Waikato, Private Bag 3105, Hamilton, New Zealand \\ 2 Sydney Institute for Astronomy, School of Physics, The University of Sydney, NSW 2006, Australia \\ Received 2014 October 16; accepted 2014 December 29; published 2015 February 20
}

\begin{abstract}
Disintegration of sunspots (and starspots) by fluxtube erosion, originally proposed by Simon and Leighton, is considered. A moving boundary problem is formulated for a nonlinear diffusion equation that describes the sunspot magnetic field profile. Explicit expressions for the sunspot decay rate and lifetime by turbulent erosion are derived analytically and verified numerically. A parabolic decay law for the sunspot area is obtained. For moderate sunspot magnetic field strengths, the predicted decay rate agrees with the results obtained by Petrovay and Moreno-Insertis. The new analytical and numerical solutions significantly improve the quantitative description of sunspot and starspot decay by turbulent erosion.
\end{abstract}

Key words: diffusion - stars: magnetic field - starspots - Sun: magnetic fields - sunspots - turbulence

\section{INTRODUCTION}

Bumba (1963) investigated how the areas of large, slowly decaying sunspots decrease with time. His data analysis suggested that the sunspot area $A$ decreases linearly with time $t$ :

$$
A(t)=A_{0}-\dot{A} t,
$$

where the decay rate $\dot{A}$ is a constant. The result is consistent with the Gnevyshev-Waldmeier relation $T \sim A_{0}$, where $T$ is the sunspot lifetime and $A_{0}$ is its initial area (see, e.g., Petrovay \& van Driel-Gesztelyi 1997 for a review). Following Bumba (1963), sunspot observations were usually interpreted in terms of the linear decay law for the sunspot area (e.g., Robinson \& Boice 1982). Yet it is difficult to distinguish linear and nonlinear decays observationally, and observations have also been interpreted using a parabolic decay law, with $A(t)$ a decreasing quadratic function of time (Moreno-Insertis \& Vázquez 1988; Martínez Pillet et al. 1993).

On the theoretical side, Meyer et al. (1974) argued that the linear decay law is a consequence of turbulent diffusion of the magnetic field across the whole area of a sunspot and expressed the constant decay rate in terms of a constant uniform diffusivity (see also Krause \& Rüdiger 1975).

Simon \& Leighton (1964) inferred from observations that the gradual disintegration of sunspots is due to "erosion" of the penumbral boundaries by supergranular flows, which occurs when bits of magnetic field are sliced away from the edges of the sunspot and swept to the supergranular cell boundaries. In contrast to the model of Meyer et al. (1974), such erosion can occur if the turbulent diffusivity associated with the flows is suppressed within the spot (Petrovay \& Moreno-Insertis 1997). Alternative theoretical approaches were reviewed by Solanki (2003).

Petrovay \& Moreno-Insertis (1997) developed the turbulent erosion model mathematically, taking into account the dependence of the turbulent diffusivity on the magnetic field strength. The diffusivity rapidly decreases if the magnetic field exceeds an energy equipartition value (Kitchatinov et al. 1994). As a result, a current sheet is formed around the spot. The model leads to the parabolic decay law, specified by a constant inward speed $w$ of the current sheet, viz.,

$$
A(t)=\pi\left(r_{0}-w t\right)^{2}
$$

for a circular flux tube (sunspot) of an initial area $A_{0}=\pi r_{0}^{2}$. Moreover, the model yields $w \sim 1 / r_{0}$, and so it agrees with the Gnevyshev-Waldmeier relation. Petrovay \& Moreno-Insertis (1997) concluded that solar observations are consistent with turbulent erosion based on a granule-size diffusion length. Petrovay \& van Driel-Gesztelyi (1997) presented observational evidence in favor of the parabolic decay rate, predicted by the turbulent erosion model, although an independent magnetohydrodynamic simulation suggested that the sunspot decay law is almost linear (Rüdiger \& Kitchatinov 2000). Petrovay et al. (1999) also explored the effect of a preexisting "plage" field on the decay rate, whereas Chatterjee et al. (2006) applied the model to the development of twist in a flux tube rising through the solar convection zone.

The analytical results of the turbulent erosion model have been recently used to complement numerical simulations of sunspot formation and decay (e.g., Rempel \& Cheung 2014). The model has also been applied to starspots, with a goal of using the starspot decay data to place constraints on the magnetic diffusivity, which may be useful for dynamo models (e.g., Strassmeier 2009; Bradshaw \& Hartigan 2014).

It is worthwhile to revisit the turbulent erosion model of sunspot decay. The original calculation of Petrovay \& MorenoInsertis (1997) was guided by numerical results and onedimensional analytical solutions. A dimensional argument was used to estimate the magnetic field gradient at the sunspot edge:

$$
\frac{\partial B}{\partial r} \sim-\frac{B_{e}}{r_{0}},
$$

where $B_{e}$ is the magnetic field value above which the turbulent diffusivity is assumed to be suppressed (see Equations (11)-(16) in Petrovay \& Moreno-Insertis 1997). In addition, their numerical estimate for the sunspot lifetime appears to be based on an estimate of the current sheet speed $w$ rather than on direct computation.

A rigorous derivation of the sunspot decay law is necessary if the theory is to be used to develop reliable predictive tools. Explicit analytical predictions of the turbulent erosion model could complement more detailed numerical (e.g., Hurlburt \& DeRosa 2008) and empirical (Gafeira et al. 2014) models of sunspot decay. Hence our aim is to put the turbulent erosion model on a firmer footing. We do this by formulating a moving 
boundary problem (Carslaw \& Jaeger 1959; Crank 1984) for the model and solving it to derive a prediction for the sunspot decay law. In the remainder of the paper, we present the new analytical (Section 2) and numerical (Section 3) results and their discussion (Section 4).

\section{FORMULATION OF THE PROBLEM AND ANALYTICAL RESULTS}

In order to model the turbulent erosion of a sunspot, we follow Petrovay \& Moreno-Insertis (1997) and consider the evolution of a cylindrically symmetric magnetic flux tube. The magnetic field $\mathbf{B}=B(r, t) \hat{\mathbf{z}}$ is described by the diffusion equation

$$
\frac{\partial B}{\partial t}=\frac{1}{r} \frac{\partial}{\partial r}\left(r D \frac{\partial B}{\partial r}\right) .
$$

Here $t$ is time, and $r$ is the distance from the $z$-axis.

The turbulent diffusivity $D=D(B)$ is strongly suppressed when the magnetic field exceeds an energy equipartition value $B_{e}=\sqrt{4 \pi \rho} u$ where $\rho$ is the mass density and $u$ is a characteristic turbulent speed (e.g., Kitchatinov et al. 1994). For instance, taking a photospheric value of $\rho \approx 2 \times 10^{-7} \mathrm{~g} \mathrm{~cm}^{-3}$ and a granular value of $u \approx 2 \times 10^{5} \mathrm{~cm} \mathrm{~s}^{-1}$ yields $B_{e} \approx 400 \mathrm{G}$ (Petrovay \& Moreno-Insertis 1997). To simplify the analytical treatment, we assume

$$
D(B)=D_{0}=\text { const }, \quad B<B_{e}
$$

and

$$
D(B)=0, \quad B>B_{e} .
$$

The initial value problem is specified by the field profile

$$
B(r, 0)=B_{0}=\text { const, } \quad 0<r<r_{0},
$$

and $B(r, 0)=0$ otherwise (see Tlatov \& Pevtsov 2014 for recent data on sunspot magnetic fields). We nondimensionalize the problem by measuring the magnetic fields, times, and distances in units of $B_{e}, r_{0}^{2} / D_{0}$, and $r_{0}$, respectively.

The sunspot size decreases with time because the magnetic flux is removed by diffusion. The strongly nonlinear dependence of the diffusivity $D$ on the magnetic field strength leads to the formation of a tangential discontinuity at the edge $r=r_{e}$ of the flux tube. Physically, the magnetic field discontinuity at $r_{e}(t)$ corresponds to a current sheet at the sunspot edge, where the magnetic flux removal is made possible by a strongly localized electric current.

It is useful to observe that the problem at hand is mathematically similar to a moving boundary problem in the theory of heat conduction, and so we can use existing methods of analysis. In particular, an analog of the Stefan condition is obtained by the integration of the governing diffusion equation across the moving boundary $r=r_{e}(t)$ (Carslaw \& Jaeger 1959). Allowing for the tangential discontinuity at $r=r_{e}(t)$, we substitute

$$
B(r, t)=B_{0}+\left(B-B_{0}\right) H\left[r-r_{e}(t)\right],
$$

where $H$ is the Heaviside step function, into Equation (4) and integrate across the discontinuity (from $r_{e}-0$ to $r_{e}+0$ ). The result is

$$
\left(B_{0}-1\right) \frac{d r_{e}}{d t}=\left.\frac{\partial B}{\partial r}\right|_{r=r_{e}+0},
$$

where we used $B\left(r_{e}-0, t\right)=B_{0}, B\left(r_{e}+0, t\right)=1$, and $D\left(r_{e}-0\right)=0$.
To find an approximate analytical solution, we use the pseudosteady-state approximation that can be adopted when the rate of change $\dot{r}_{e}$ is small compared with a global diffusion rate $\sim 1$, making it possible to neglect the term $\partial B / \partial t$ in Equation (4). Physically, the magnetic field profile near a moving boundary relaxes to a pseudo-steady state on a time scale $\delta t_{D} \simeq(\delta r)^{2} / D$ where $\delta r \simeq \dot{r}_{e} \delta t$ is the displacement of the boundary $r_{e}(t)$ in a time $\delta t$. The approximation is valid if the relaxation is sufficiently rapid, say if $\delta t_{D} \ll \delta t$. In our dimensionless variables, we have $\delta r \simeq r_{e} \leqslant 1$ and $D=1$, and so $\delta t_{D} / \delta t \simeq \dot{r}_{e}$. If $T$ is the sunspot lifetime, we use $\dot{r}_{e} \simeq T^{-1}$ to infer that, as long as $T \gg 1$, the approximation is globally valid in the range $1<t<T-1$. We show below that roughly $T \simeq B_{0}-1$. Consequently, the pseudo-steady-state approximation becomes more accurate as $B_{0}$ increases. Detailed analysis of the accuracy of the approximation can be found in standard textbooks on heat conduction (e.g., Crank 1984; Hill \& Dewynne 1987).

Inside the spot, the vanishing diffusivity implies that the magnetic field is constant:

$$
B\left(r<r_{e}(t), t\right)=B_{0} .
$$

Outside the spot, the pseudo-steady-state field satisfies

$$
\frac{1}{r} \frac{\partial}{\partial r}\left(r \frac{\partial B}{\partial r}\right)=0,
$$

and so

$$
r \frac{\partial B}{\partial r}=\text { const. }
$$

Equation (5) gives the boundary condition

$$
B\left(r=r_{e}(t), t\right)=1,
$$

which would be $B=B_{e}$ in dimensional units. The magnetic field diffusion outside the spot causes the field to become negligibly small at some $r=r_{f}(t)$ outside the spot. Solutions of the standard diffusion equation in two dimensions suggest that $r_{f}(t)=(2 t)^{1 / 2}$ (Carslaw \& Jaeger 1959). Thus we set

$$
B\left(r=r_{f}(t), t\right)=0 .
$$

The solution of Equation (12), satisfying the boundary conditions at $r_{e}$ and $r_{f}$, is given by

$$
B\left(r>r_{e}(t), t\right)=\frac{\ln \left(r^{2} / 2 t\right)}{\ln \left(r_{e}^{2} / 2 t\right)} .
$$

On substituting this into Equation (9), we get

$$
\left(B_{0}-1\right) \frac{d r_{e}^{2}}{d t}=\frac{4}{\ln \left(r_{e}^{2} / 2 t\right)} \text {. }
$$

We also obtained a similar differential equation for $r_{e}(t)$ using an independent heat-balance approximation (e.g., Crank 1984). We do not present the results here: although the approach requires longer calculations, it does not appear to be more accurate than the pseudo-steady-state approximation.

Equation (16) does not appear to have a solution in elementary functions. The magnitude of its right-hand side is of order unity, which yields an order-of-magnitude estimate $r_{e}^{2}(t) \simeq$ $1-t /\left(B_{0}-1\right)$. Consequently, we have $T \simeq B_{0}-1$ and $r_{e}^{2}(T / 2) \simeq$ $1 / 2$. Next we obtain a more accurate solution of Equation (16). An approximate polynomial solution would be convenient 
for comparison with the available observational results and theoretical predictions. We use a quadratic approximation:

$$
r_{e}^{2}(t) \approx c_{0}+c_{1}(t-T / 2)+c_{2}(t-T / 2)^{2},
$$

where

$$
c_{1}=\left.\frac{d r_{e}^{2}}{d t}\right|_{t=T / 2}
$$

and

$$
c_{2}=\left.\frac{1}{2} \frac{d^{2} r_{e}^{2}}{d t^{2}}\right|_{t=T / 2}
$$

We expand $r_{e}^{2}(t)$ about $t=T / 2$ because this is where the pseudo-steady-state approximation is expected to be most accurate. The remaining constants $c_{0}$ and $T$ are defined by the conditions

$$
r_{e}^{2}(0)=1
$$

and

$$
r_{e}^{2}(T)=0
$$

Equations (17), (20), and (21) give

$$
r_{e}^{2}(t) \approx 1+\left(c_{1}-c_{2} T\right) t+c_{2} t^{2} .
$$

Here

$$
T=-\frac{1}{c_{1}}
$$

is the sunspot lifetime, unless $T^{\prime}<T$ where

$$
T^{\prime}=-\frac{c_{1}}{c_{2}}
$$

is the other root of the equation $r_{e}^{2}(t)=0$.

We evaluate the constant $c_{1}$ by substituting the order-ofmagnitude estimates $t=T / 2 \simeq\left(B_{0}-1\right) / 2$ and $r_{e}^{2}(T / 2) \simeq 1 / 2$ into Equation (16). This yields an accurate expression for $c_{1}$ because $T / 2$ and $r_{e}^{2}(T / 2)$ only appear in the argument of the logarithm in Equation (16). The resulting prediction for the sunspot lifetime is as follows:

$$
T=\frac{1}{4}\left(B_{0}-1\right) \ln 2\left(B_{0}-1\right),
$$

which should be compared with Equation (16) in Petrovay \& Moreno-Insertis (1997) for the inward speed $w=-\dot{r}_{e}$ of the current sheet. In their model, $w=$ const and the sunspot lifetime is given by $T_{\mathrm{PM}}=r_{e} / w$, which leads to

$$
T_{\mathrm{PM}}=2^{1 / 3} B_{0}
$$

in our dimensionless variables. The same result (up to a numerical coefficient) is obtained by nondimensionalizing our Equation (3), substituting it into Equation (9), and assuming $\dot{r}_{e}=$ const.

Differentiation of Equation (16) with respect to time yields

$$
c_{2}=\left.\frac{2}{\left(B_{0}-1\right)\left[\ln \left(r_{e}^{2} / 2 t\right)\right]^{2}}\left[\frac{1}{t}-\frac{4}{\left(B_{0}-1\right) r_{e}^{2} \ln \left(r_{e}^{2} / 2 t\right)}\right]\right|_{t=T / 2} .
$$

Again using $t=T / 2 \simeq\left(B_{0}-1\right) / 2$ and $r_{e}^{2}(T / 2) \simeq 1 / 2$ is justified when these quantities appear in the argument of the logarithm. Therefore,

$$
c_{2}=\frac{1}{\ln 2\left(B_{0}-1\right)}\left[1+\frac{1}{2 r_{e}^{2}(T / 2)}\right] \frac{1}{T^{2}},
$$

where $T$ is defined by Equation (25). The solution below can be used to verify that $r_{e}^{2}(T / 2)=1 / 2+O\left(1 / \ln 2\left(B_{0}-1\right)\right)$. Thus using $r_{e}^{2}(T / 2) \simeq 1 / 2$ in Equation (28) only leads to a relatively small error of order $1 /\left[T \ln 2\left(B_{0}-1\right)\right]^{2}$, and we get

$$
c_{2} \approx \frac{2}{T^{2} \ln 2\left(B_{0}-1\right)} .
$$

Collecting the results, we obtain a parabolic decay law for the sunspot area:

$$
r_{e}^{2}(t) \approx 1-\left(1+\frac{2}{\ln 2\left(B_{0}-1\right)}\right) \frac{t}{T}+\frac{2}{\ln 2\left(B_{0}-1\right)} \frac{t^{2}}{T^{2}}
$$

The sunspot lifetime is given by $T$ in Equation (25) if $B_{0}>B_{*}$ and by

$$
T^{\prime}=\frac{1}{8}\left(B_{0}-1\right)\left[\ln 2\left(B_{0}-1\right)\right]^{2}
$$

if $1<B_{0}<B_{*}$, where

$$
B_{*}=1+e^{2} / 2 \approx 4.7
$$

corresponds to $T=T^{\prime}$.

Our explicit analytical solution for $r_{e}(t)$ provides an improved quantitative description of sunspot decay by turbulent erosion. Notably, if $B_{0}=B_{*}$, our solution predicts a constant decrease rate $w=2 /\left(B_{*}-1\right) \approx 0.54$ of the fluxtube radius:

$$
r_{e}(t) \approx 1-w t
$$

as in the parabolic decay law, predicted by Petrovay \& MorenoInsertis (1997). More generally, we obtain a constant speed approximation

$$
w \approx\left(\frac{1}{2}+\frac{1}{\ln 2\left(B_{0}-1\right)}\right) \frac{1}{T}
$$

by defining $w=-\dot{r}_{e}(0)$ in our solution. If $A(t)=\pi r_{e}^{2}$ is the sunspot area, the accuracy of the approximation can be quantified by calculating

$$
\left.\frac{2 \ddot{A}}{\dot{A}^{2}}\right|_{t=0}=\frac{8 \ln 2\left(B_{0}-1\right)}{\left[2+\ln 2\left(B_{0}-1\right)\right]^{2}},
$$

which would be unity in the model of Petrovay \& MorenoInsertis (1997).

On returning to the original dimensional quantities, we get the lifetime-size scaling $T \sim A_{0}$, where $A_{0}=\pi r_{0}^{2}$ is the initial cross-sectional area of the flux tube. This result formally agrees with the Gnevyshev-Waldmeier relation for sunspot lifetimes. It is worth stressing that the statistical nature of the relation should follow from the strong dependence of $T$ on the spot magnetic field $B_{0}$.

\section{NUMERICAL RESULTS}

The analytical results obtained in Section 2 may be tested by numerical solution of Equation (4). Following Petrovay \& Moreno-Insertis (1997), we assume the analytical forms for the 


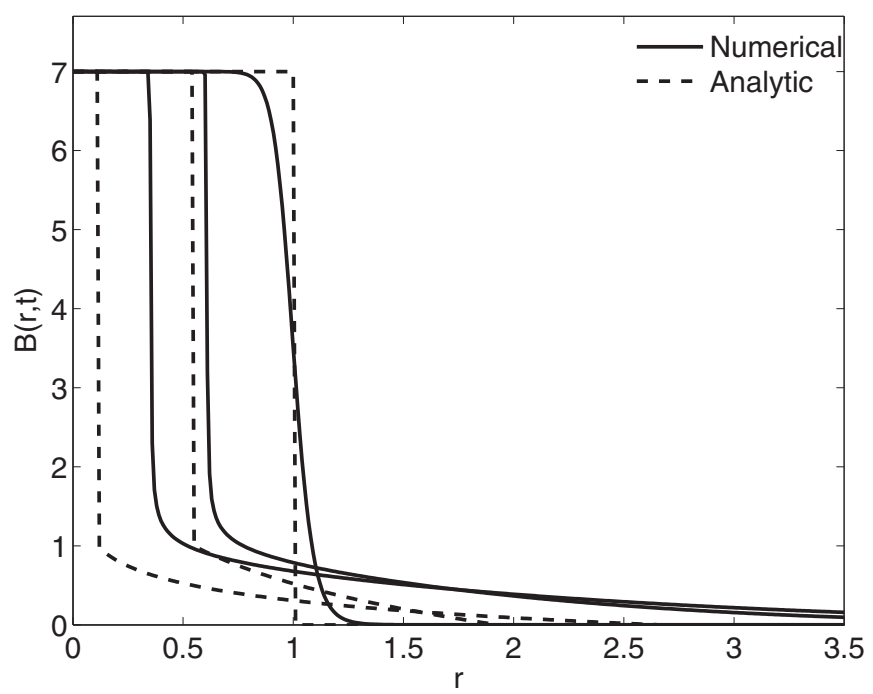

Figure 1. Magnetic field vs. radius at times $t=0, t=0.5 T$, and $t=0.95 T$, for the case $B_{0}=7$. The solid curves show the numerical solutions (for the parameter choices $\alpha_{D}=7, \alpha_{B}=22$ ), and the dashed curves show the analytical solutions.

diffusivity and the initial field profile:

$$
\begin{aligned}
& D(B)=\frac{1}{1+|B|^{\alpha_{D}}}, \\
& B(r, 0)=\frac{B_{0}}{1+r^{\alpha_{B}}},
\end{aligned}
$$

where we use the nondimensionalization introduced in Section 2. The parameter $\alpha_{D}$ in Equation (36) determines the strength of the suppression of diffusion by the field, and the parameter $\alpha_{B}$ in Equation (37) specifies the initial spot profile. In the following we choose $\alpha_{B}=22$ to model an isolated flux tube with nearly constant internal field strength, and $\alpha_{D}=7$, to represent strong suppression of diffusion. For the purpose of numerical solution, the radius of the spot at time $t$ is defined by the condition

$$
B\left(r_{e}, t\right)=\frac{1}{2} B_{0}
$$

We solve Equation (4) using a Crank-Nicolson scheme (e.g., Press et al. 1992) which is described in the Appendix. The diffusion equation is evolved in time in the region $0 \leqslant r \leqslant r_{m}$ with the boundary condition $\partial B / \partial r=0$ at $r=0$ and with a boundary condition at $r=r_{m}$ which allows loss of flux from the region. Note that Petrovay \& Moreno-Insertis (1997) used a less realistic condition $\partial B / \partial r=0$ at an outer boundary (at $r=10$ ), and their numerical solution was based on a Lax-Wendroff scheme.

Figure 1 illustrates the numerical solution for the case $B_{0}=7$. The solid curves in the figure show the numerical result for $B(r, t)$ as a function of $r$ for times $t=0, t=0.5 T$, and $t=0.95 T$, where $T$ is the analytical decay time, defined by Equation (25). The solutions are shown for the region $r \leqslant 1 / 2 r_{m}$, where $r_{m}=7$ is the outer boundary of the numerical domain. Figure 1 also shows the analytical solution at the same times, following Equations (10) and (15) with the spot radius defined by Equation (30). The spot decays more rapidly in the analytical solution, and the magnetic field outside the spot decreases more rapidly with increasing radius. The numerical solutions illustrate how the initial central flux concentration is redistributed to larger radius by diffusion, leading to an initial

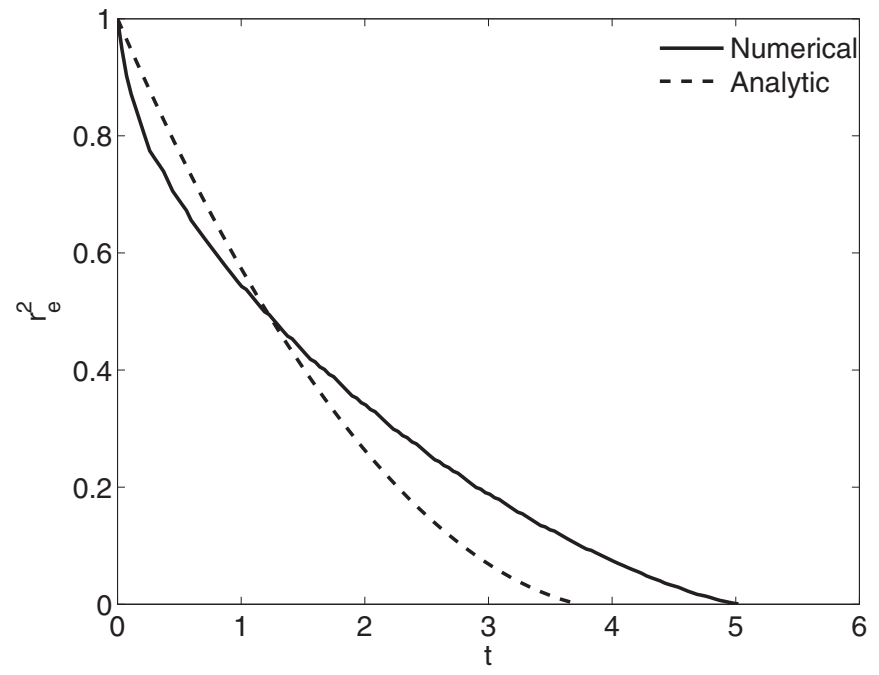

Figure 2. Sunspot radius squared vs. time, for the case shown in Figure 1. The solid curve shows the numerical solution, and the dashed curve shows the analytical solution.

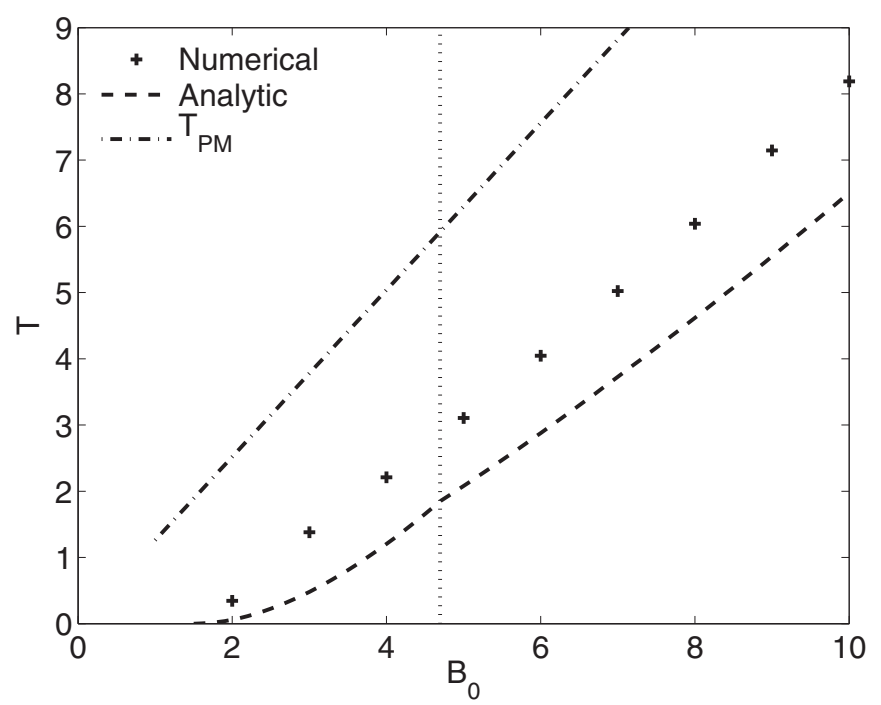

Figure 3. Decay time vs. sunspot field strength $B_{0}$. The crosses indicate results for numerical solutions (with the parameters $\alpha_{D}=7, \alpha_{B}=22$ ), and the dashed curve is the analytical solution of this paper. The dotted vertical line indicates which of two times ( $T$ and $\left.T^{\prime}\right)$ applies. The dot-dashed line is the decay time for the Petrovay \& Moreno-Insertis (1997) model (our Equation (26)).

increase in field strength at points external to the spot. The qualitative behavior of the numerical solution is generally well reproduced by the analytical solution.

Figure 2 shows the square of the sunspot radius as a function of time for the same case $B_{0}=7$. The solid curve shows the numerical solution, with $r_{e}$ defined by Equation (38), and the dashed curve shows the analytical solution defined by Equation (30). The analytical solution decays more rapidly than the numerical solution, but both clearly show the departure from a linear decay law. The analytical estimate for the decay time is $T=3.72$, and the numerical decay time is 5.02 .

Figure 3 plots numerically determined sunspot decay times versus central field strength $B_{0}$ (crosses). The decay time is seen to depend almost linearly on field strength. The dashed curve shows the analytical results of Section 2 . Recall that Equation (30) defines two times at which $r_{e}(t)=0$, namely $T$ in Equation (25) and $T^{\prime}$ in Equation (31). The decay time for the spot is given by $T$ if $B_{0}>B_{*}$, and by $T^{\prime}$ if $B_{0}<B_{*}$, 


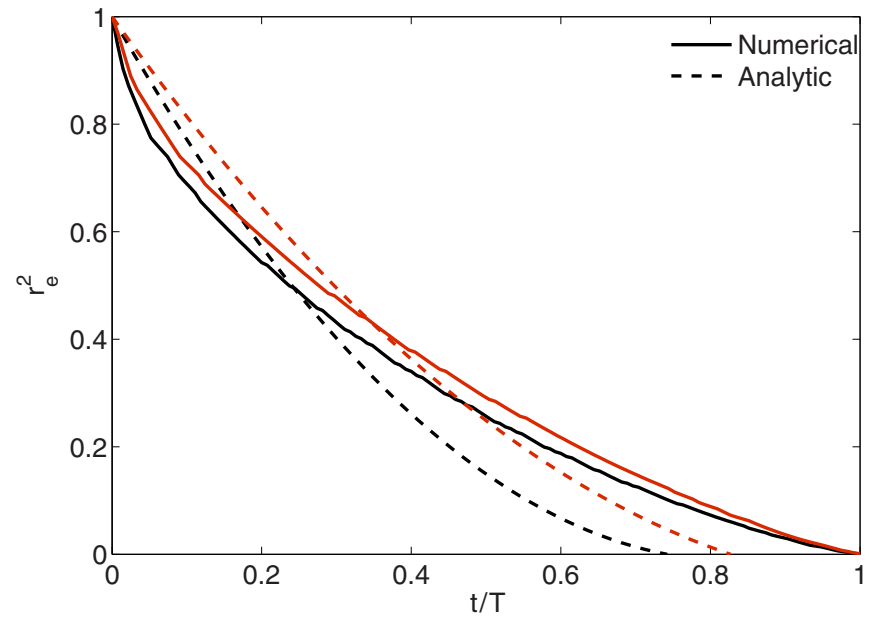

Figure 4. Sunspot radius squared vs. time, normalized by the decay time $T$ from the numerical solution, for $B_{0}=7$ (black) and $B_{0}=14$ (red). Other parameters are as in Figure 1. The solid curves show the numerical solution, and the dashed curves show the analytical solution.

where $B_{*}$ is defined by Equation (32). The dotted vertical line in Figure 3 indicates the threshold value $B_{*}$. Figure 3 also shows the decay time in the Petrovay \& Moreno-Insertis (1997) model. Our analytical predictions agree with the numerical results: in particular the rates of increase of decay time with $B_{0}$ are quite similar. Although our analytical model underestimates the decay times, it is significantly more accurate than the earlier model.

Finally, we emphasize that our calculation generally yields a time-dependent rate of decrease of the fluxtube radius $r_{e}(t)$. Equation (30) predicts that the deviation from the parabolic decay law, derived by Petrovay \& Moreno-Insertis (1997), should increase as the initial magnetic field $B_{0}$ increases. As a result, a linear decay law (rather than a parabolic one) should become more accurate as $B_{0}$ increases, although the logarithmic dependence on $B_{0}$ makes the effect rather weak. Figure 4 shows the effect of doubling the field strength $B_{0}$ on the shape of the function $r_{e}^{2}(t)$. While the computation time and numerical errors increase for larger $B_{0}$, we do see numerical evidence that the decay law becomes more linear for a larger initial magnetic field, which is consistent with our analytical prediction.

\section{DISCUSSION}

We have presented in this paper a quantitative theory of sunspot decay by turbulent erosion, considered as a moving boundary problem. The physical mechanism of sunspot erosion was proposed by Simon \& Leighton (1964), and a sunspot decay law due to turbulent erosion was derived by Petrovay \& Moreno-Insertis (1997; see also Petrovay et al. 1999, and references therein). Although Petrovay and collaborators correctly identified the key dependence of the decay rate on the sunspot magnetic field $B_{0}$, the accuracy of the analytical predictions was limited: for instance, we have shown that the numerically computed sunspot lifetime is about a half of that predicted.

Our Equation (25) for the sunspot decay time $T$ is an improvement on Equation (26), derived by Petrovay \& MorenoInsertis (1997). Equation (30) confirms that the decay law for the sunspot area $A(t)=\pi r_{e}^{2}$ is in general parabolic, as long as higher-order terms in $t / T$ can be neglected. Equation (30) also quantifies the accuracy of the assumption, made by Petrovay \& Moreno-Insertis (1997), that the inward speed $\dot{r}_{e}$ of the current sheet surrounding the decaying spot is constant. We have shown that the assumption is justified if the initial sunspot magnetic field $B_{0}$ is not too large. Equation (30) predicts that a linear decay law should become more accurate as $B_{0}$ increases. The numerical solutions in Figure 4 confirm this prediction, although, as noted by the referee, they also show that the deviation from a linear decay is systematically underestimated in the analytical model.

Application of the turbulent erosion theory to sunspot and starspot decay is a topic of current research interest (e.g., Strassmeier 2009; Rempel \& Cheung 2014; Bradshaw \& Hartigan 2014), and so our quantitative analytical predictions, reinforced by numerical solutions, should be useful in studies of solar and stellar activity. The value of an analytical calculation is that it can be used to verify more detailed magnetohydrodynamic simulations (e.g., Hurlburt \& DeRosa 2008; Rempel \& Cheung 2014) and to guide empirical models (e.g., Gafeira et al. 2014).

The erosion model can be further refined. For instance, we assumed $D_{0}=$ const in our analysis of Section 2. The rate of relative diffusion of two photospheric magnetic fragments is controlled by turbulent eddies whose size is equal to the current distance between the fragments. Consequently, the turbulent diffusivity is expected to be scale-dependent. In practice the turbulent diffusivity is determined by applying the induction equation to pairs of solar magnetograms (e.g., Chae et al. 2008, and references therein). Scale-dependent turbulent diffusivity has been invoked to interpret observations of photospheric flux cancellation (Litvinenko 2011) and the dispersion of photospheric bright points (Abramenko et al. 2011). The turbulent erosion model of sunspot decay should be generalized to incorporate the dependence of the effective diffusivity on the size of a decaying sunspot. In addition, although Petrovay \& MorenoInsertis (1997) argued that regular radial flows play little if any role in sunspot decay, the effect of regular photospheric flows on sunspot decay should be investigated in more detail. Finally, recent observations emphasized the difference between the maximum and average sunspot magnetic field strengths (Tlatov \& Pevtsov 2014), and so it may be worthwhile to derive a solution for a more general initial profile of the magnetic field within the sunspot, as well as a more realistic dependence of the turbulent diffusivity on the field strength within the spot.

The authors thank the referee for comments and suggestions that helped to improve the original manuscript.

\section{APPENDIX \\ NUMERICAL METHOD}

The numerical solutions in Section 3 use the Crank-Nicolson method to solve the nonlinear diffusion Equation (4), in which a discrete version of the equation is linearized at each time step. The Crank-Nicolson method is a preferred one for solution of parabolic partial differential equations because it is unconditionally stable, and second order accurate in time (e.g., Press et al. 1992).

Equation (4) is solved at spatial locations $r_{j}=(j-1) h$ with $j=1,2, \ldots, L$ and $h=r_{m} /(L-1)$, for a sequence of times $t_{n}=(n-1) \tau$, with $n=1,2, \ldots$. Introducing the notation $B_{j}^{n}=B\left(r_{j}, t_{n}\right)$ and $D_{j}^{n}=D\left(B_{j}^{n}\right)$, we consider a Crank-Nicolson scheme with the differencing of terms in Equation (4):

$$
\left.r \frac{\partial B}{\partial t}\right|_{t_{n}, r_{j}} \approx r_{j} \frac{B_{j}^{n+1}-B_{j}^{n}}{\tau}
$$


and

$$
\begin{aligned}
& \left.\left.\frac{\partial}{\partial r}\left[r D(B) \frac{\partial B}{\partial r}\right]\right|_{t_{n}, r_{j}} \approx \frac{1}{2} \frac{\partial}{\partial r}\left[r D(B) \frac{\partial B}{\partial r}\right]\right|_{t_{n+1}, r_{j}}+\left.\frac{1}{2} \frac{\partial}{\partial r}\left[r D(B) \frac{\partial B}{\partial r}\right]\right|_{t_{n}, r_{j}} \\
& \approx \frac{1}{2 h}\left(r_{j+\frac{1}{2}} D_{j+\frac{1}{2}}^{n+1} \frac{B_{j+1}^{n+1}-B_{j}^{n+1}}{h}-r_{j-\frac{1}{2}} D_{j-\frac{1}{2}}^{n+1} \frac{B_{j}^{n+1}-B_{j-1}^{n+1}}{h}\right) \\
& +\frac{1}{2 h}\left(r_{j+\frac{1}{2}} D_{j+\frac{1}{2}} D_{j+1}^{n}-B_{j}^{n}-r_{j-\frac{1}{2}} D_{j-\frac{1}{2}}^{n+1} \frac{B_{j}^{n}-B_{j-1}^{n}}{h}\right) .
\end{aligned}
$$

In the final expression in Equation (A2), the centered differences are taken about locations $r_{j-\frac{1}{2}}$ and $r_{j+\frac{1}{2}}$. We introduce the approximations $D_{j}^{n+1} \rightarrow D_{j}^{n}$ and

$$
D_{j \pm \frac{1}{2}}^{n} \rightarrow D_{j \pm}^{n}=\frac{1}{2}\left(D_{j}^{n}+D_{j \pm 1}^{n}\right)
$$

involving a linearization in time and a spatial averaging respectively. Combining Equations (A1) and (A2) we have

$$
B_{j}^{n+1}-\frac{s}{2(j-1)} F\left(B_{j}^{n+1}\right)=B_{j}^{n}+\frac{s}{2(j-1)} F\left(B_{j}^{n}\right),
$$

with $s=\tau / h^{2}$ and

$$
\begin{aligned}
F\left(B_{j}^{n}\right)= & \left(j-\frac{1}{2}\right) D_{j+}^{n} B_{j+1}^{n}-\left[\left(j-\frac{1}{2}\right) D_{j+}^{n}+\left(j-\frac{3}{2}\right) D_{j-}^{n}\right] \\
& \times B_{j}^{n}+\left(j-\frac{3}{2}\right) D_{j-}^{n} B_{j-1}^{n} .
\end{aligned}
$$

A von Neumann analysis of Equations (A4)-(A5) in the linear case $D_{j}=D_{0}=$ const confirms that the scheme is unconditionally stable. The corresponding explicit scheme with the same spatial differencing is unstable if $D_{0} \tau / h^{2}>1 / 2$ (e.g., Press et al. 1992).

Equations (A4) and (A5) define the update for points $j=$ $2,3, \ldots, L-1$. At the point $j=1$, the boundary condition $\partial B /\left.\partial r\right|_{r=0}=0$ is enforced using the one-sided second order difference approximation to the derivative:

$$
\left.\frac{\partial B}{\partial r}\right|_{t_{n+1}, r_{1}} \approx \frac{-3 B_{1}^{n+1}+4 B_{2}^{n+1}-B_{3}^{n+1}}{2 h}=0,
$$

or

$$
-3 B_{1}^{n+1}+4 B_{2}^{n+1}-B_{3}^{n+1}=0 .
$$

For the point $j=L$ we obtain an update equation allowing flux transport across the boundary $r=r_{m}$ via a discretization of Equation (4) at time $t=t_{n}$ and spatial location $r=r_{L-1 / 2}$ with differencing schemes

$$
\left.r \frac{\partial B}{\partial t}\right|_{t_{n}, r_{L-\frac{1}{2}}} \approx r_{L-\frac{1}{2}} \frac{B_{L-\frac{1}{2}}^{n+1}-B_{L-\frac{1}{2}}^{n}}{\tau}
$$

and

$$
\begin{gathered}
\left.\frac{\partial}{\partial r}\left[r D \frac{\partial B}{\partial r}\right]\right|_{t_{n}, r} \approx \frac{1}{h}\left(\left.r_{L} D_{L}^{n} \frac{\partial B}{\partial r}\right|_{t_{n}, r_{L}}-\left.r_{L-1} D_{L-1}^{n} \frac{\partial B}{\partial r}\right|_{t_{n}, r_{L-1}}\right) \\
\approx \frac{1}{h}\left(r_{L} D_{L}^{n} \frac{B_{L-2}^{n}-4 B_{L-1}^{n}+3 B_{L}^{n}}{2 h}-r_{L-1} D_{L-1}^{n} \frac{B_{L}^{n}-B_{L-2}^{n}}{2 h}\right),
\end{gathered}
$$

where Equation (A9) involves the one-sided second order difference approximation to the derivative:

$$
\left.\frac{\partial B}{\partial r}\right|_{t_{n}, r_{L}} \approx \frac{B_{L-2}^{n}-4 B_{L-1}^{n}+3 B_{L}^{n}}{2 h} .
$$

Equations (A8) and (A9) give the update equation for $j=L$ :

$$
\begin{aligned}
B_{L}^{n+1}+B_{L-1}^{n+1}= & s \\
& \left(\frac{L-2}{L-\frac{3}{2}} D_{L-1}^{n}+\frac{L-1}{L-\frac{3}{2}} D_{L}^{n}\right) B_{L-2}^{n} \\
& +\left(1-4 s \frac{L-1}{L-\frac{3}{2}} D_{L}^{n}\right) B_{L-1}^{n} \\
& +\left(1-s \frac{L-2}{L-\frac{3}{2}} D_{L-1}^{n}+3 s \frac{L-1}{L-\frac{3}{2}} D_{L}^{n}\right) B_{L}^{n}
\end{aligned}
$$

Equations (A4), (A5), (A7), and (A11) provide a system of linear equations for the field values $B_{j}^{n+1}$, with $j=1,2, \ldots, L$, which must be solved at each time step. The scheme may be written in matrix form as

$$
\left(\mathrm{I}^{\prime}+a_{-} \mathrm{A}\right) \mathbf{B}^{n+1}=\left(\mathrm{I}^{\prime}+a_{+} \mathrm{A}^{\prime}\right) \mathbf{B}^{n},
$$

where $\mathbf{B}^{n}=\left(B_{1}^{n}, B_{2}^{n}, \ldots, B_{L}^{n}\right)^{T}, a_{ \pm}=\mp \frac{1}{2} s, \mathrm{I}^{\prime}$ is the $L \times L$ matrix

$$
\mathrm{I}^{\prime}=\operatorname{diag}(0,1, \ldots, 1),
$$

the matrix $\mathrm{A}$ is defined by $A_{L j}=0$ for all $j$ except

$$
A_{L L-1}=\frac{1}{a_{-}}
$$

and the matrix $\mathrm{A}^{\prime}$ is defined by $A_{L j}^{\prime}=0$ for all $j$ except

$$
\begin{aligned}
A_{L L-2}^{\prime} & =\frac{s}{a_{+}}\left(\frac{L-2}{L-\frac{3}{2}} D_{L-1}^{n}+\frac{L-1}{L-\frac{3}{2}} D_{L}^{n}\right), \\
A_{L L-1}^{\prime} & =\left(1-4 s \frac{L-1}{L-\frac{3}{2}} D_{L}^{n}\right), \\
A_{L L}^{\prime} & =\frac{s}{a_{+}}\left(-\frac{L-2}{L-\frac{3}{2}} D_{L-1}^{n}+3 \frac{L-1}{L-\frac{3}{2}} D_{L}^{n}\right) .
\end{aligned}
$$

A simple test for the new method is provided by the exact solution for constant diffusivity $D_{0}$ with a Gaussian profile:

$$
B(r, t)=\frac{\Phi_{0}}{\sigma^{2}} \exp \left(-\frac{1}{2} r^{2} / \sigma^{2}\right)
$$

with

$$
\sigma^{2}=2 D_{0} t+\sigma_{0}^{2},
$$

where the constant $\sigma_{0}$ defines the initial width. The magnetic flux (divided by $2 \pi$ ) from $r=0$ to $r=r_{m}$ for this solution is

$$
\begin{aligned}
\Phi\left(r_{m}, t\right) & =\int_{0}^{r_{m}} r B(r, t) d r \\
& =\Phi_{0}\left[1-\exp \left(-\frac{1}{2} r_{m}^{2} / \sigma^{2}\right)\right] .
\end{aligned}
$$

Equation (A18) provides a check on the implementation of the boundary condition at $r=r_{m}$. The method incurs truncation 
error (proportional to $\tau^{2}$ and $h^{2}$ ) at each time step, and the accumulation of the error limits the accuracy of the solution when the system is evolved over many time steps. The calculations presented in this paper are checked by trials with different spatial steps.

\section{REFERENCES}

Abramenko, V. I., Carbone, V., Yurchyshyn, V., et al. 2011, ApJ, 743, 133

Bradshaw, S. J., \& Hartigan, P. 2014, ApJ, 795, 79

Bumba, V. 1963, BAICz, 14, 91

Carslaw, H. S., \& Jaeger, J. C. 1959, Conduction of Heat in Solids (Oxford: Clarendon Press)

Chae, J., Litvinenko, Y. E., \& Sakurai, T. 2008, ApJ, 683, 1153

Chatterjee, P., Choudhuri, A. R., \& Petrovay, K. 2006, A\&A, 449, 781

Crank, J. 1984, Free and Moving Boundary Problems (Oxford: Clarendon Press)

Gafeira, R., Fonte, C. C., Pais, M. A., \& Fernandes, J. 2014, SoPh, 289, 1531

Hill, J. M., \& Dewynne, J. N. 1987, Heat Conduction (Oxford: Blackwell Scientific)

Hurlburt, N., \& DeRosa, M. 2008, ApJL, 684, L123
Kitchatinov, L. L., Pipin, V. V., \& Rüdiger, G. 1994, AN, 315, 157

Krause, F., \& Rüdiger, G. 1975, SoPh, 42, 107

Litvinenko, Y. E. 2011, ApJL, 731, L39

Martínez Pillet, V., Moreno-Insertis, F., \& Vázquez, M. 1993, A\&A, 274, 521

Meyer, F., Schmidt, H. U., Weiss, N. O., \& Wilson, P. R. 1974, MNRAS, 169,35

Moreno-Insertis, F., \& Vázquez, M. 1988, A\&A, 205, 289

Petrovay, K., Martínez Pillet, V., \& van Driel-Gesztelyi, L. 1999, SoPh, 188,315

Petrovay, K., \& Moreno-Insertis, F. 1997, ApJ, 485, 398

Petrovay, K., \& van Driel-Gesztelyi, L. 1997, SoPh, 176, 249

Press, W. H., Flannery, B. P., Teukolsky, S. A., \& Vetterling, W. T. 1992, Numerical Recipes in C: The Art of Scientific Computing (2nd ed.; Cambridge: Cambridge Univ. Press)

Rempel, M., \& Cheung, M. C. M. 2014, ApJ, 785, 90

Robinson, R. D., \& Boice, D. C. 1982, SoPh, 81, 25

Rüdiger, G., \& Kitchatinov, L. L. 2000, AN, 321, 75

Simon, G. W., \& Leighton, R. B. 1964, ApJ, 140, 1120

Solanki, S. K. 2003, A\&ARv, 11, 153

Strassmeier, K. G. 2009, A\&ARv, 17, 251

Tlatov, A. G., \& Pevtsov, A. A. 2014, SoPh, 289, 1143 